\title{
Generation of iPS-derived
}

\section{model cells for analyses of hair shaft differentiation}

\author{
Takumi Kido, Tomoatsu Horigome, Minori Uda, Naoki Adachi, \\ and Yohei Hirai \\ Department of Biomedical Chemistry, Graduate School of Science \\ and Technology, Kwansei Gakuin University, Sanda 669-1337, Japan
}

BioTechniques 63:131-134 (September 2017) doi 10.2144/000114589

Keywords: hair shaft; iPS; screening; model cell; keratin; differentiation; bone morphogenic protein-4

Supplementary material for this article is available at www.BioTechniques.com/article/114589.

Biological evaluation of hair growth/differentiation activity in vitro has been a formidable challenge, primarily due to the lack of relevant model cell systems. To solve this problem, we generated a stable model cell line in which successive differentiation via epidermal progenitors to hair components is easily inducible and traceable. Mouse induced pluripotent stem (iPS) cell-derived cells were selected to stably express a tetracycline (Tet)-inducible bone morphogenic protein-4 (BMP4) expression cassette and a luciferase reporter driven by a hair-specific keratin 31 gene (krt31) promoter (Tet-BMP4-KRT31-Luc IPS). While TetBMP4-KRT31-Luc iPS cells could be maintained as stable iPS cells, the cells differentiated to produce luciferase luminescence in the presence of all-trans retinoic acid (RA) and doxycycline (Dox), and addition of a hair differentiation factor significantly increased luciferase fluorescence. Thus, this cell line may provide a reliable cell-based screening system to evaluate drug candidates for hair differentiation activity.

While hair loss is a problem for many people, effective therapeutic agents for this condition are quite limited $(1,2)$. Intensive efforts toward developing therapeutic chemical compounds have been made by analyzing the mechanisms underlying hair follicle development, regeneration, and induction of the telogen-to-anagen transition in mouse models $(3,4)$. Currently, stumptailed macaques (5) or organ culture systems (6) have also been used to develop and test remedies for hair loss. However, these models are cost/timeconsuming, and the results are often complicated by inter-individual differences. Given that differentiation of epidermal progenitors into hair follicle components would be an important process regardless of the assay system, it is conceivable that compounds with effective trichogenic activity could be initially screened for this process in a reliable model cell line system. To date, however, there are no effective cell culture models, since primary keratinocytes are mortal and suffer lot-to-lot variation. In addition, all available keratinocyte cell lines have lost the ability to differentiate into hair components. Recently, epidermal progenitor cells generated from pluripotent stem cells have garnered special attention as a tool to screen drugs possessing trichogenic activity (7). However, these progenitor cells are unstable and prone to differentiation into not only hairs/hair follicles but also several epidermal lineages (8). Based on this, we attempted to prepare gene-manipulated mouse induced pluripotent stem (iPS) cells that could be stably maintained but also undergo effective and traceable differentiation into hair shaft cells upon exposure to simple stimuli.

Pluripotent stem cells undergo triploblastic differentiation, with ectodermal cells capable of subsequent differentiation into neuronal and epidermal lineages. In this process, retinoic acid (RA) plays an important role in ectodermal differentiation, and the locally high concentration of bone morphogenic protein-4 (BMP4) affects subsequent differentiation into epidermal progenitors $(9,10)$ (Figure 1A). While treatment with RA can be easily done, addition of recombinant BMP4 is costly, and sustained expression of BMP4 might lead to an unstable cellular context in embryonic stem (ES)/iPS cells. Thus, using a recently established technique (11), we prepared iPS-derived stable cells that express exogenous BMP4 upon treatment with tetracycline (Tet) or doxycycline (Dox) (Figure 1B); we named these cells Tet-BMP4 iPS cells. Although precise determination of the local concentration of exogenously expressed BMP4 may not be feasible, Tet-BMP4 iPS cells activated downstream elements of the BMP4 signaling pathway within several hours (Figure 1B). On the other hand, expression of BMP4 and the activation of its signaling pathway were undetectable if the cell culture medium did not contain Dox. In response to

\section{METHOD SUMMARY}

To prepare simple and reliable model cells for evaluating hair differentiation activity, mouse induced pluripotent stem (iPS) cells were selected to stably express tetracycline (tet)-inducible bone morphogenic protein-4 (BMP4) expression cassette and a luciferase reporter gene driven by the hair-specific keratin 31 (krt31) gene promoter. 
stimulation with Dox and RA, Tet-BMP4 iPS cells changed to nanog-negative differentiated cells, including krt14positive epidermal progenitors, even in the absence of embryoid body formation (Figure 1, C and D). Unexpectedly, the KRT14-positive cell population was still very limited $(<10 \%$; Supplementary Figure S1), and a considerable number of gata4-positive endodermal cells, but not brachury $(T)$-positive mesodermal cells, were also generated by this treatment (Figure 1C). Furthermore, krt14-positive epidermal progenitors spontaneously differentiated into transglutaminase 1 gene (tgase1)-positive keratinocytes within a few days (Figure 1D). Consistently, the expression of $k r t 75$, a marker for the hair medulla and the innermost layer (companion layer) of the outer root sheath (ORS) of hair follicles, was increased, followed by synchronous up-regulation of very specific markers for the hair cortex, krt31 and krt33a (Figure $1 D$ ), which is reminiscent of hair follicle development in vivo (12). It is noteworthy that the mRNA expression of these hair/ hair follicle markers decreased after 3-4 days of treatment (Figure 1D), probably because highly keratinized cells lose their nuclei and cease mRNA production, whereas endodermal subpopulations may continue to grow and express their mRNA. We noticed that keratinocyte growth factor (KGF) increased the number of krt14-positive cell populations; however, subsequent differentiation toward hair/hair follicle components was not accelerated, implying that KGF increased the number of epidermal progenitors but subsequently directed them to differentiate into skin epidermal cells (Supplementary Figure S2). On the other hand, co-culture with dermal papilla cells (DPCs), which reportedly secrete trichogenic factors (13), up-regulated both krt31 and krt75, and stimulation with wnt5a, a DPC-derived factor for hair follicle morphogenesis (14), led to up-regulation of krt75 in Tet-BMP4-iPS cells (Figure 2A).

Given that treatment with RA and Dox together generated endodermal subpopulations from Tet-BMP4-iPS cells, which grew without exhibiting hair-differentiation potential, and that differentiated keratinocytes from a limited number of epidermal progenitors began losing mRNA-producing ability, additional gene


Figure 1. Tetracycline (Tet)-bone morphogenic protein-4 (BMP4) induced pluripotent stem (iPS) cells can differentiate into hair components upon treatment with retinoic acid (RA) and doxycycline (Dox). (A) Schematic diagram of the differentiation of pluripotent stem cells. Embryonic stem (ES)/iPS cells treated with RA preferentially differentiate into ectodermal cells that subsequently differentiate into krt14positive epidermal progenitors in response to a high concentration of BMP4. Keratinocytes for stratified epidermis and hair/hair follicle components -including outer root sheath (ORS), inner root sheath (IRS), and hair - derive from the epidermal progenitors. Nestin: marker for neuronal progenitors. (B) Generation of Tet-BMP4 iPS cells. Mouse iPS cells (iPS-MEF-Ng-20D-17) were stably introduced with PiggyBac-Tet transposon plasmid together with the expression plasmids for reverse tetracycline-controlled transactivator (rtTA) and transposase (Supplementary Materials and Methods). These cells (Tet-BMP4 iPS cells) produced detectable amounts of BMP4 (48 kDa; lower left gel) and activated Smad1/5/8, downstream elements of the BMP4 signaling pathway within several hours after Dox treatment $(5 \mu \mathrm{g} / \mathrm{mL})$. $\beta$-actin: loading control. (C) The upper two graphs show quantitative RT-PCR analyses of mRNA expression of ectodermal lineage markers in Tet-BMP4 iPS cells cultured for 9 h. krt14: marker for epidermal progenitors. nestin: marker for neuronal progenitors. The lower three graphs show quantitative RT-PCR analyses of mRNA expression of markers for undifferentiated cells (nanog), mesodermal lineages $(T$ ), and endodermal lineages (gata4) in the cells cultured for $24 \mathrm{~h}$. Open, dotted, and closed bars represent relative mRNA expression in the cells cultured without RA/Dox, with RA only, and with RA/Dox, respectively. mRNA expression was normalized to that of $\beta$-actin. (D) Upper left is a schematic diagram of the epidermis and hair follicles. Transglutaminase 1 (TGase1) is a marker for differentiated keratinocytes in the stratified epidermis, outer root sheath (ORS), and inner root sheath (IRS). KRT31 and KRT33 are highly specific markers for hair cortex. KRT75 is a marker for the innermost layer (companion layer) of the ORS and hair medulla. The lower left and right graphs show daily expression of krt14, tgasel, krt31, krt33a, and krt75 in Tet-BMP4 iPS cells cultured with RA and Dox. The number of krt14-positive epidermal progenitors was initially increased, then decreased and replaced by that of tgase1-positive differentiated keratinocytes (left). The expression of krt31, krt33a, and krt75 changed in accordance with keratinocyte differentiation (right). 
A
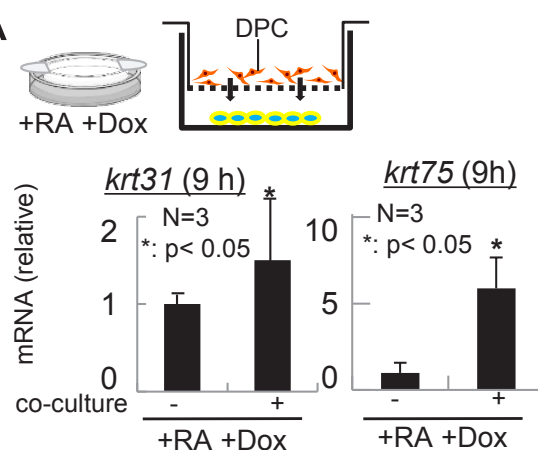

B
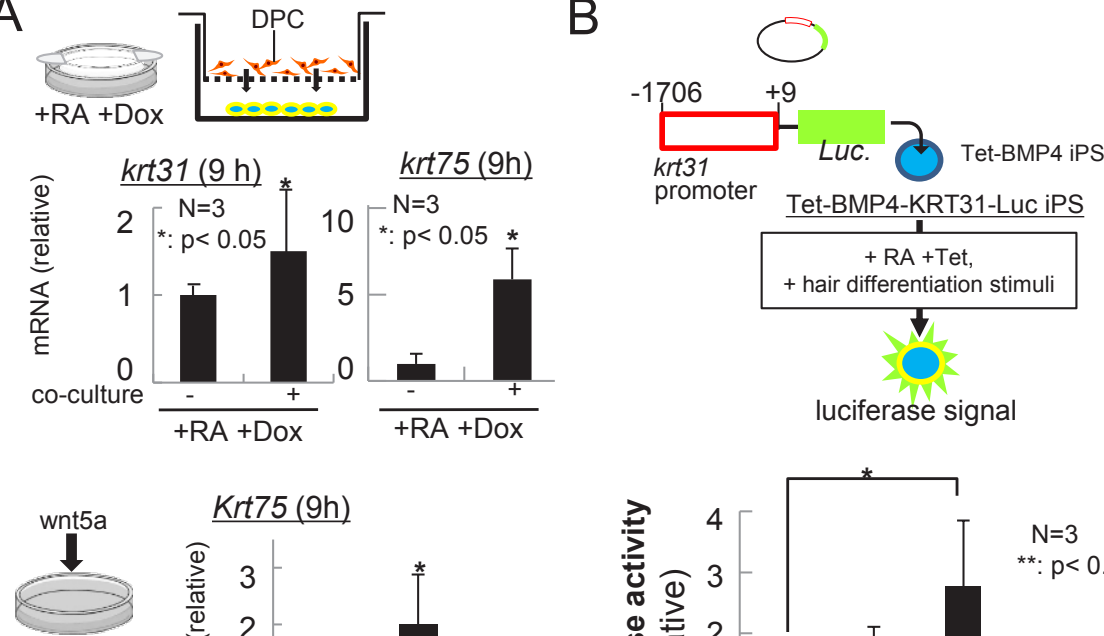

$+\mathrm{RA}+\mathrm{Dox}$

$\mathrm{N}=7$

**: $p<0.05$

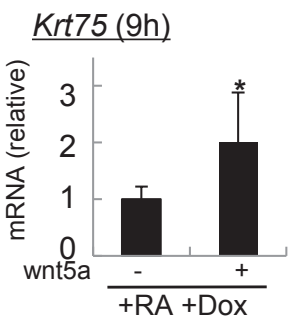

C

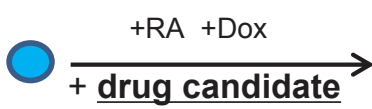

Tet-BMP4-KRT31-Luc iPS

(maintainable as

normal iPS cells)
Figure 2. Detection of trichogenic activity in tetracycline (Tet)-bone morphogenic protein-4 (BMP-4) induced pluripotent stem (iPS) cells. (A) Upper: Factors secreted from dermal papilla cells (DPCs) significantly accelerate the expression of krt31 and krt75 in Tet-BMP4 iPS cells cultured with retinoic acid (RA) and doxycycline (Dox) for 9 h. A tissue culture insert (Falcon) onto which DPCs have been cultured for 1 day was placed onto Tet-BMP4 iPS cells in 1 well of a 24-well plate, which allowed Tet-BMP4 iPS cells to receive soluble factors from DPCs without direct cell-cell contact. Lower: Wnt5a, which is a DPC-produced hair growth factor, significantly up-regulated krt75 in Tet-BMP4 iPS cells cultured with RA and Dox. (B) Introduction of the luciferase gene under control of the krt31 promoter enables simple and easy evaluation of possible trichogenic activities. Upper: Schematic diagram of the generation of Tet-BMP4KRT31-Luc iPS cells. A luciferase reporter plasmid containing the krt31 promoter (Supplementary Figure S3) was introduced into Tet-BMP4 iPS cells (Tet-BMP4-KRT31-Luc iPS). Lower: Relative luciferase activity in Tet-BMP4-KRT31-Luc iPS cells treated with soluble factors from DPCs using the co-culture system where medium contained RA and Dox. A significant increase in luciferase activity was detected within several hours. Rapid decrease in luciferase activity after $16 \mathrm{~h}$ implies the dramatic acceleration of hair shaft differentiation/denucleation. (C) Summary of the simple assay system established in this study. Tet-BMP4-KRT31-Luc iPS cells cultured with RA and Dox produce luminescence within several hours when treated simultaneously with reagents having trichogenic activity. manipulation was carried out to improve the sensitivity of detection of hair differentiation in this cell line system. We found that the luciferase reporter gene placed under the control of the krt31 promoter (krt31-Luc) enabled more sensitive detection of hair differentiation in these cells (Tet-BMP4-KRT31-LuciPS), since they produced significant luciferase signals within 6 hours in response to factors secreted from DPCs (Figure 2B). These results suggest that Tet-BMP4-KRT31-Luc-iPS cells, which can be stably maintained in the absence of RA and Dox, could become a reliable model system to assess hair differentiation activity when cultured with RA and Dox (Figure 2C).

While methods for the maintenance and differentiation of pluripotent stem cells are not entirely conserved among mammalian species (15), this technique is potentially applicable to human iPS cells, since they reportedly also undergo hair/hair follicle differentiation when treated with RA and BMP4 (16). Based on our findings, iPS cells engineered for the inducible expression of a factor that induces target germ layer or progenitor formation, such as BMP4 for ectodermal differentiation, and containing a reporter gene controlled by a specific promoter activated in the target cells/tissues, such as the krt31 promoter in the hair cortex, could potentially be a reliable model for evaluation of drug efficacy.

\section{Author contributions}

T.K and Y.H designed the experiments, and T.K performed most of them. T.H, M.U., and N.A assessed the data and suggested additional experiments for confirmation. Y.H and T.K. prepared the manuscript.
Novel Design Strategy For Ultrasensitive DNA Probes Test Polythiol Probes \& Microarrays developed, Hybridization \& Higly Sensitive DNA Detection a)
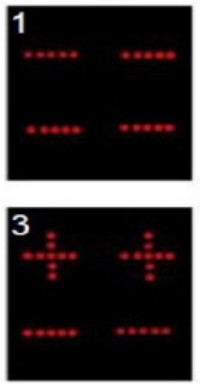

b)

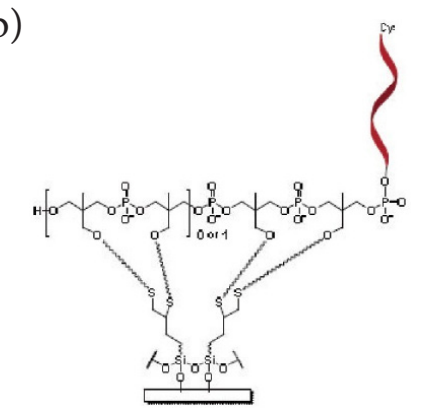

UNPARALLED Expertise in



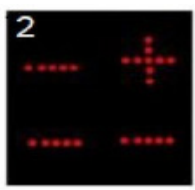

4

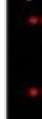

a) ried out on the plates and low level concentration of Salmonella \& Campylobacter PCR products was detected.

b). It was observed that oligos with four thiol linkages gave best results for detection of PCR amplified probes.

a1 : Blank

2 : low level of

3 : high level of

Salmonella $(1 / 10)$

Salmonella and

Campylobacter(1/60)

(1)

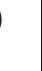

NUCLEIC ACID Chemistry since 1981

ISO 9001:2008 Certified Company

For more information on how ChemGenes
products can help you, contact us now:
t: +1 (978) 694-4500
1-800-762-9323 (USA/Canada)
w: www.ChemGenes.com
e: info@chemgenes.com




\section{Acknowledgments}

We thank all members of Hirai laboratory for helpful discussions. Part of this work was supported by the Kobayashi International Scholarship Foundation and the Ho-Yu Science Foundation (to Y. H.).

\section{Competing interests}

The authors declare no competing interests

\section{References}

1. Arif, T., K. Dorjay, M. Adil, and M. Sami. 2017. Dutasteride in androgenetic alopecia: An update. Curr. Clin. Pharmacol._12:31-35.

2. Ramdasi, S. and S.K. Tiwari. 2016. Human mesenchymal stem cell-derived conditioned media for hair regeneration applications. J. Stem Cells 11:201-211.

3. Chiang, C., R.Z. Swan, M. Grachtchouk, M. Bolinger, Y. Litingtung, E.K. Robertson, M.K. Cooper, W. Gaffield, et al. 1999. Essential role for Sonic hedgehog during hair follicle morphogenesis. Dev. Biol. 205:1-9.

4. Takebe, K., Y. Oka, D. Radisky, H. Tsuda, K. Tochigui, S. Koshida, K. Kogo, and Y. Hirai. 2003. Epimorphin acts to induce hair follicle anagen in C57BL/6 mice. FASEB J. 17:2037-2047.

5. Uno, H., A. Cappas, and C. Schlagel. 1985 Cyclic dynamics of hair follicles and the effect of minoxidil on the bald scalps of stumptailed macaques. Am. J. Dermatopathol. 7:283-297.

6. Langan, E.A., M.P. Philpott, J.E. Kloepper, and R. Paus. 2015. Human hair follicle organ culture: theory, application and perspectives. Exp. Dermatol. 24:903-911.

7. Bilousova, G., J. Chen, and D.R. Roop. 2011. Differentiation of mouse induced pluripotent stem cells into a multipotent keratinocyte lineage. J. Invest. Dermatol. 131:857-864.

8. Veraitch, O., T. Kobayashi, Y. Imaizumi, W. Akamatsu, T. Sasaki, S. Yamanaka, M. Amagai, H. Okano, and M. Ohyama. 2013. Human induced pluripotent stem cell-derived ectodermal precursor cells contribute to hair follicle morphogenesis in vivo. J. Invest. Dermatol. 133:1479-1488.

9. Liu, S., H. Zhang, and E. Duan. 2013. Epidermal development in mammals: key regulators, signals from beneath, and stem cells. Int. J. Mol. Sci. 14:10869-10895.

10. Metallo, C.M., L. Ji, J.J. de Pablo, and S.P. Palecek. 2010. Directed differentiation of human embryonic stem cells to epidermal progenitors. Methods Mol. Biol. 585:83-92.

11. Kim, S.I., F. Oceguera-Yanez, C. Sakurai, M. Nakagawa, S. Yamanaka, and K. Woltjen. 2016. Inducible Transgene Expression in Human iPS Cells Using Versatile All-in-One piggyBac Transposons. Methods Mol. Biol. 1357:111-131

12. Langbein, L., H. Yoshida, S. PraetzelWunder, D.A. Parry, and J. Schweizer. 2010. The keratins of the human beard hair medulla: the riddle in the middle. J. Invest. Dermatol. 130:55-73.

13. Morgan, B.A. 2014. The dermal papilla: an instructive niche for epithelial stem and progenitor cells in development and regeneration of the hair follicle. Cold Spring Harb. Perspect. Med. 4:a015180.

14. Hu, B., K. Lefort, W. Qiu, B.C. Nguyen, R.D. Rajaram, E. Castillo, F. He, Y. Chen, et al. 2010. Control of hair follicle cell fate by underlying mesenchyme through a CSL-Wnt5a-FoxN1 regulatory axis. Genes Dev. 24:1519-1532.

15. Nakagawa, M., M. Koyanagi, K. Tanabe, K. Takahashi, T. Ichisaka, T. Aoi, K. Okita, Y. Mochiduki, et al. 2008. Generation of induced pluripotent stem cells without Myc from mouse and human fibroblasts. Nat. Biotechnol. 26:101-106.

16. Yang, R., Y. Zheng, M. Burrows, S. Liu, Z. Wei, A. Nace, W. Guo, S. Kumar, et al. 2014 Generation of folliculogenic human epithelial stem cells from induced pluripotent stem cells. Nat. Commun. 5:3071.

Received 05 April 2017; accepted 07 August 2017.

Address correspondence to Yohei Hirai, Department of Biomedical Chemistry, Graduate School of Science and Technology, Kwansei Gakuin University. 2-1, Gakuen, Sanda 669-1337, Japan. E-mail: y-hirai@kwansei.ac.jp

To purchase reprints of this article, contact: biotechniques@fosterprinting.com

\section{RAMP-UP} cell-based assays!

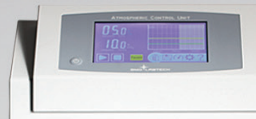

CLARIOstar

-

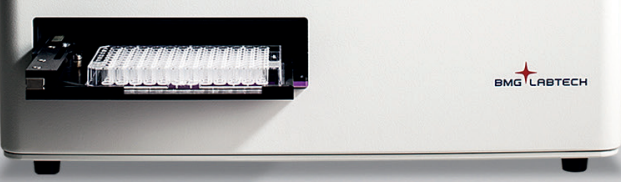

CLARIOstar ${ }^{\circledR}$ with $A C U$

Full flexibility in cellular assays with the new gas ramping function

For the first time scientists are given the capability to fully manipulate the environment within the microplate reader, by mimicking in vitro hypoxia, ischaemia/reperfusion and much more.

The CLARIOstar with LVF Monochromators ${ }^{\text {TM }}$ is not only the most-sensitive monochromator-based plate reader today. Its Atmospheric Control Unit (ACU) can run gas ramps, modifying $\mathrm{O}_{2}$ and $\mathrm{CO}_{2}$ gas levels and rapidly restoring back atmospheric conditions.

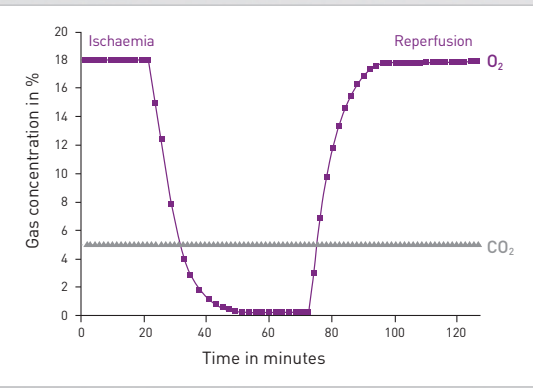

Example of $\mathrm{O}_{2}$ deprivation and reoxygenation (down to $0.2 \% \mathrm{O}_{2}$; purple) with steady $5 \% \mathrm{CO}_{2}$ (grey) performed by the CLARIOstar with ACU. 